\title{
Identification of potential prognostic long non-coding RNA signatures based on a competing endogenous RNA network in lung adenocarcinoma
}

\author{
XIAOJUAN WANG, YAWEN DING, BANGMING DA, YAN FEI and GANG FENG \\ Department of Oncology, Wuhan Fourth Hospital (Puai Hospital), Tongji Medical College, \\ Huazhong University of Science and Technology, Wuhan, Hubei 430000, P.R. China
}

Received March 16, 2018; Accepted September 3, 2018

DOI: $10.3892 /$ or.2018.6719

\begin{abstract}
A number of experimental and computational studies have demonstrated the key roles of long non-coding RNAs (lncRNAs) acting as competing endogenous RNAs (ceRNAs) in the tumorigenesis of lung adenocarcinoma (LUAC). However, there remains a requirement for prognostic candidate biomarkers acting as ceRNAs for the prediction of overall survival in patients with LUAC. The main goal of the present study was to identify novel lncRNAs associated with LUAC overall survival and assess their prognostic values. The study analyzed coding RNA and ncRNA expression profiles of patients with LUAC by retrieving existing RNA-sequencing datasets from The Cancer Genome Atlas database, and 2,507 differentially expressed mRNAs, 1,633 lncRNAs and 113 miRNAs were screened from patients with LUAC compared with those of adjacent normal samples $(\mathrm{P}<0.01$ and $\mid \log \mathrm{FCl}>2)$. Of these LUAC-specific RNAs, 134 lncRNAs, 21 miRNAs and 34 mRNAs were used to build an IncRNA-mRNA-miRNA ceRNA network, among which 8 lncRNAs and 9 mRNAs were associated with overall survival in patients with LUAC by acting as ceRNAs. Next, an IncRNA-based prognostic signature was constructed by risk scoring approach based on the expression levels of 9 prognosis-associated lncRNAs using Cox's regression analysis. Moreover, the prognostic capacity of the 9-lncRNA signature was independent of known clinical prognostic factors. These results provide novel insight into
\end{abstract}

Correspondence to: Dr Gang Feng, Department of Oncology, Wuhan Fourth Hospital (Puai Hospital), Tongji Medical College, Huazhong University of Science and Technology, 473 Hanzheng Street, Wuhan, Hubei 430000, P.R. China

E-mail: drfengg@hotmail.com

Abbreviations: ncRNA, non-coding RNA; ceRNA, competing endogenous RNA; LUAC, lung adenocarcinoma; ROC, receiver operating characteristic; TCGA, The Cancer Genome Atlas

Keywords: lung adenocarcinoma, long non-coding RNAs, competing endogenous RNA network, overall survival, prognostic signature the potential of IncRNA ceRNAs to be candidate biomarkers associated with LUAC overall survival.

\section{Introduction}

Lung adenocarcinoma (LUAC) is the leading cause of cancer-associated mortality worldwide and is one major subtype of non-small cell lung cancer, defined by distinct pathological characteristics, including mixed subtype, acinar, papillary and lepidic patterns, and the solid predominant subtype with mucin production $(1,2)$. As the most common type of lung cancer, accounting for $40 \%$ of all non-small cell lung cancer cases as determined by the World Health Organization in 2012, the incidence of LUAC is on the rise mainly in women and non-smokers $(3,4)$. The 5 -year overall survival rate is $\sim 15 \%$, but has not improved in recent years. Since approximately two-thirds of LUAC patients are diagnosed at advanced cancer stages, and local or distant tumor recurrence can frequently present following surgical resection, the prognosis is poor for the majority of patients. Therefore, identifying LUAC at earlier pathological stages can greatly reduce overall mortality rates. Given that adenocarcinoma is more difficult to detect by clinical approaches, including bronchoscopy, sputum cytology and computed tomography, the major obstacle in LUAC management is the lack of an adequate method for its early detection and prognosis.

Non-coding RNAs (ncRNAs) have become increasingly relevant targets of study due to their specialized and well-adapted biological roles in tumor development (5). Generally, ncRNAs can be divided into two major classes based on their size: Small ncRNAs and long ncRNAs (lncRNAs). Small ncRNAs consist of several subtypes, including microRNAs (miRNAs/miRs), ribosomal RNAs, small nucleolar RNAs and transfer ribonucleic acids (6). An ever-increasing body of evidence demonstrates the key role of miRNAs in tumor biology contributing to tumorigenesis by modulating oncogenic and tumor suppressor pathways (7-9). However, research on lncRNAs is in its infancy compared with miRNA research. Importantly, lncRNAs have been implicated in several biological processes from pluripotency to immune responses, and are predicted to be involved in more complex mechanisms such as tumor regulation $(10,11)$. One 
of the best-studied lncRNAs, X-inactive specific transcript, is involved in the development of several cancer types through recruitment of chromatin-modifying complexes to inactivate an entire chromosome in the majority of cells (12).

Since ncRNAs serve various important roles in tumor development, interactions between miRNAs and lncRNAs have become an area of focus for the identification of putative ncRNA biomarkers for tumor prognosis. As our understanding of the transcriptome space has expanded and the development of RNA-sequencing technology has taken place, a novel hypothesis known as the competing endogenous RNA (ceRNA) hypothesis has emerged in recent years $(13,14)$. One lncRNA, hepatocellular carcinoma upregulated lncRNA, has been shown to be one of the most clearly overexpressed ncRNAs in hepatocellular carcinoma, and contains miR-372-binding sites to reduce miR-372 expression and activity (15). Another IncRNA and ceRNA, papillary thyroid carcinoma susceptibility candidate 3 , has been identified to be downregulated in thyroid cancer and mediates the expression of miR-574-5p (16). In addition to IncRNA ceRNAs, certain miRNAs and mRNAs also have ceRNA capacity. Several lncRNA ceRNAs have been found to be involved in the diagnosis and prognosis of patients with lung tumors $(17,18)$. Nevertheless, the prognostic value of lncRNA ceRNAs in LUAC has not yet been fully investigated.

In the current study, to identify LUAC-specific lncRNAs involved in ceRNA crosstalk, RNA-sequencing data and clinical data were obtained from The Cancer Genome Atlas (TCGA) database and an IncRNA-mRNA-miRNA ceRNA network was constructed. Combined with survival analysis, analyses of these data identified a 9-IncRNA signature (LASiglnc-9) with prognostic value to predict overall survival in patients with LUAC.

\section{Materials and methods}

Data source and patient information. All RNA expression data and patient clinical data were obtained from TCGA Data Portal (https://portal.gdc.cancer.gov), which is open-access and publicly available. LUAC-related RNA-sequencing data were downloaded with the key words 'lung adenocarcinoma' and 'RNA-seq'. A total of 594 LUAC patients were included and sample exclusion criteria were follows: i) Patients who were not histologically diagnosed with LUAC; ii) patients who suffered from one or more malignancies besides LUAC; and iii) samples without complete data. Gene expression profiles for 535 tumor samples and 59 adjacent non-tumor samples, and miRNA expression data for 521 LUAC samples and 46 adjacent normal samples were obtained. In addition, clinical data for 482 LUAC patients, including 260 male and 222 female patients, were also downloaded from TCGA Data Coordinating Center. There were 170 patients with lymphatic metastasis and 312 patients with non-lymphatic metastasis. Additionally, 164 patients presented with distant organ metastases and 318 patients presented with non-distant metastasis. Patients were classified as stage I-II (well and moderately differentiated LUAC, $n=377$ ) and stage III-IV (poorly differentiated LUAC, $n=105$ ) according to guidelines from the Union for International Cancer Control (19).
Differential expression analysis of LUAC data. To determine the differential expression of mRNAs, lncRNAs and miRNAs between tumor and adjacent normal tissues in LUAC samples, a Bioconductor package edgeR (version 3.6) (20) was used for the gene differential expression analysis. A P-value of $<0.01$ and $\mid \log \mathrm{FCl}>2$ were set as the cut-off criteria. Volcano plots were drawn using gplots package (version 3.0.1; http://cran.r-project. org/web/packages/gplots/index.html). Heatmaps were constructed using pheatmap package (version 1.0.8; http://cran.r-project.org/web/packages/pheatmap/index.html).

ceRNA network construction and functional annotation. Considering the important role of interactions between lncRNAs, mRNAs and miRNAs in tumorigenesis and development, the ceRNA networks of LUAC were constructed based on three steps: i) LUAC-specific lncRNAs with an absolute $\mathrm{P}$-value of $<0.01$ and $\mid \log \mathrm{FCl}>2$ were retained; ii) miRcode online tool (http://www.mircode.org) was applied to predict potential target miRNAs of differentially expressed lncRNAs and to predict lncRNA-miRNA interactions; and iii) potential mRNAs targeted by miRNAs were retrieved from miRDB (http://www.mirdb.org/index.html), miRTarBase (http://mirtarbase.mbc.nctu.edu.tw/php/index.php) and TargetScan (http://www.targetscan.org/vert_71/). Finally, miRNAs that are negatively regulated by lncRNAs and mRNAs were selected to construct the ceRNA network. To visualize the lncRNA-mRNA-miRNA ceRNA network, cytoscape v3.5.1 (21) was used for network construction. To further study the biological roles of differentially expressed mRNAs targeted by lncRNAs and miRNAs in the ceRNA network, the Database for Annotation, Visualization and Integrated Discovery (DAVID, http://david.abcc.ncifcrf.gov/) was used. Kyoto Encyclopedia of Genes and Genomes (KEGG) and Gene Ontology (GO) biological processes were annotated at significance levels of $\mathrm{P}<0.05$.

Survival analysis. Kaplan-Meier survival analysis and a log-rank test were used to evaluate the association between expression levels of differentially expressed mRNAs, lncRNAs and miRNAs in the ceRNA network and the overall survival of the patients. To obtain more detail on the role of lncRNAs in LUAC, the univariate Cox's proportional hazards regression model with a significant level set at 0.01 was applied to analyze differentially expressed lncRNAs and mRNAs from the ceRNA network that were associated with overall survival. Next, the selected differentially expressed mRNAs and IncRNAs were fit into a multivariate Cox regression analysis to build the lncRNA-based prognostic signature and lncRNA-mRNA-based prognostic signature. The prognostic risk score for predicting overall survival was calculated using the following formula: Risk score $=\exp _{1} * \beta_{1}+\exp _{2} * \beta_{2}+\ldots+$ $\exp _{n} * \beta_{n}$, where exp indicates expression level and $\beta$ is the regression coefficient. The linear combination of expression levels of LUAC-specific mRNAs or lncRNAs with estimated regression coefficients was obtained from the aforementioned multivariate Cox regression analysis (22). LUAC patients were divided into high-risk and low-risk groups using the median risk score ( 0.959 for the LASiglnc- 9 signature; 0.923 for the LASiglnc2-m3 signature). The time-dependent receiver operating characteristic (ROC) curves were drawn using the 
A

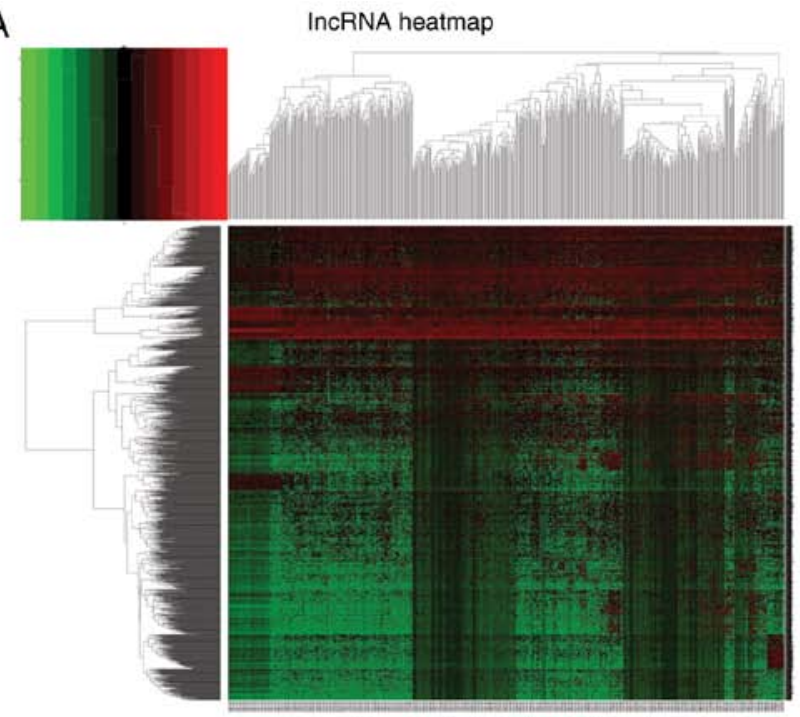

miRNA heatmap

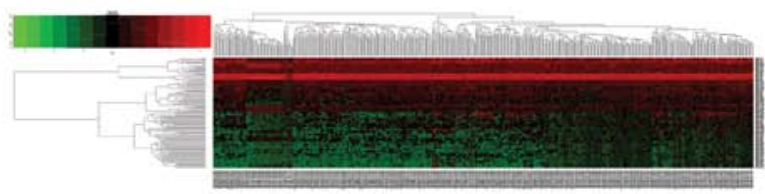

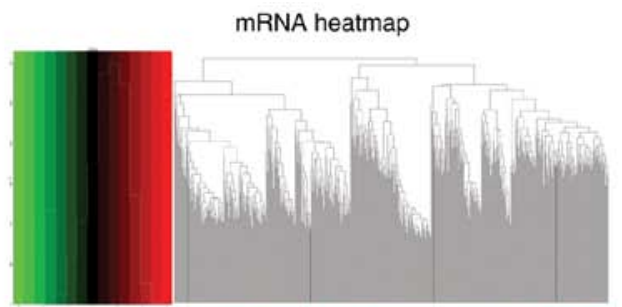

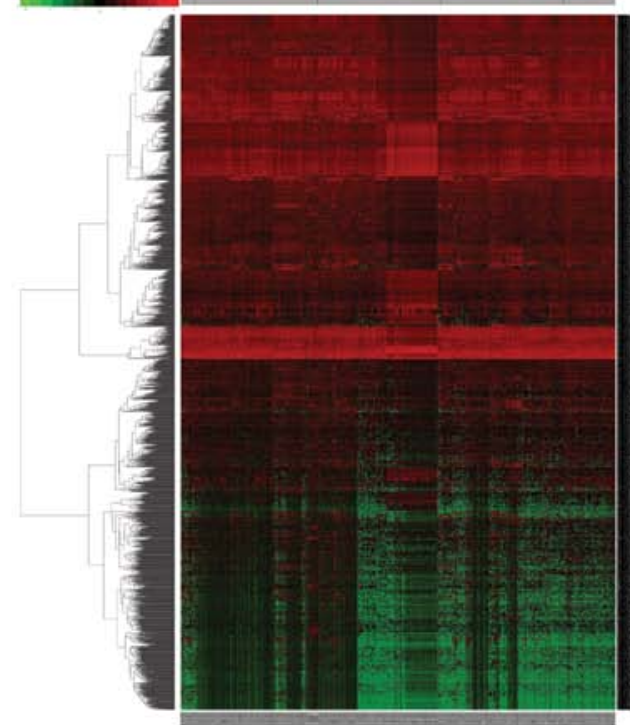

B
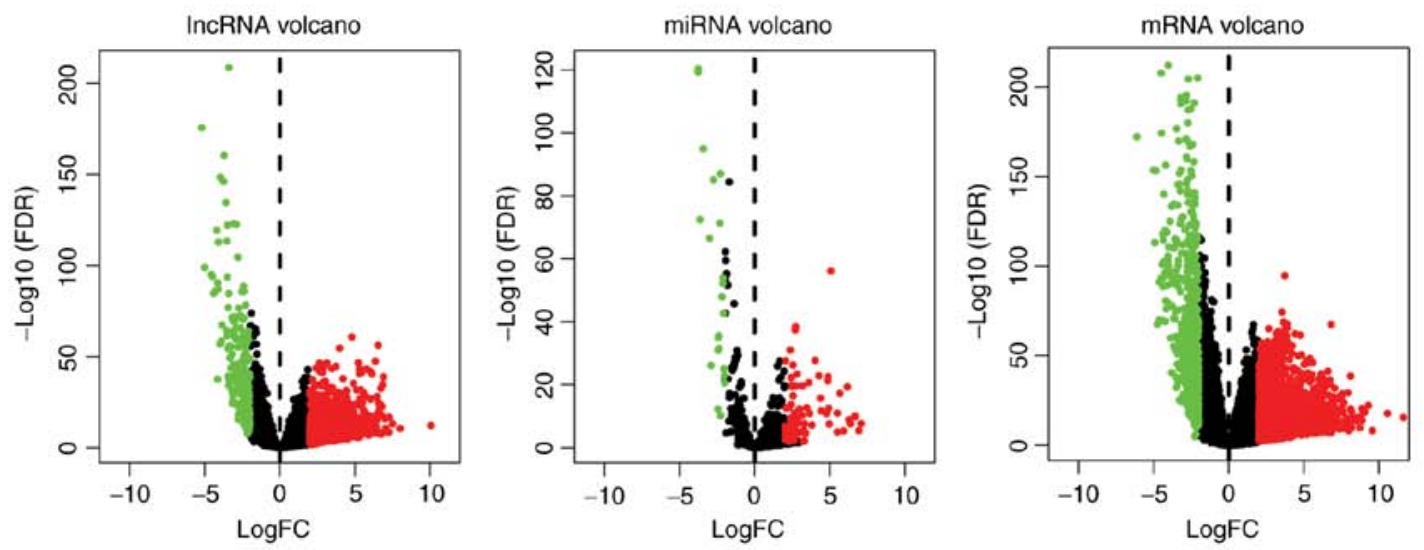

Figure 1. Heat maps and volcano plots of differentially expressed lncRNAs, mRNAs and miRNAs in patients with LUAC. (A) The hierarchical clustering heat maps of differentially expressed lncRNAs, mRNAs and miRNAs between LUAC and adjacent normal samples. (B) Volcano plot of LUAC-specific lncRNAs, mRNAs and miRNAs. lncRNA, long non-coding RNA; miRNA, microRNA; LUAC, lung adenocarcinoma; FDR, false discovery rate; FC, fold-change.

$\mathrm{R}$ package 'survival-ROC' to compare the specificity and sensitivity of the risk prediction of the survival rate for specific lncRNAs and mRNAs in the model. Meanwhile, univariate and multivariate Cox's analyses were applied for prognostic prediction of risk score and clinical features, including age, gender, stage of pathology and Tumor-Node-Metastasis (TNM) staging system (23). Hazard ratios (HRs) and 95\% confidence intervals (CIs) were assessed using the Cox regression model. All statistical analyses were conducted with R software (version 3.4.1).

\section{Results}

Identification of differentially expressed RNAs in LUAC from RNA-seq data. In the present study, RNA-seq data, including gene and miRNA expression data, was retrieved from TCGA data portal for the purpose of finding biomarkers associated with tumor prognosis. Compared with adjacent normal samples, the LUAC samples contained a total of 2,507 differentially expressed mRNAs (1,977 upregulated and 527 downregulated mRNAs), 1,633 differentially expressed lncRNAs (1,425 upregulated and 208 downregulated lncRNAs) and 113 differentially expressed miRNAs (88 upregulated and 23 downregulated miRNAs). The differentially expressed lncRNAs, miRNAs and mRNAs showed clear separation in the heat maps (Fig. 1A) and volcano plots (Fig. 1B).

miRNA target prediction and ceRNA network. To predict the lncRNAs targeted by miRNAs, the miRcode online tool was used and 134 lncRNAs, including 115 upregulated and 19 downregulated lncRNAs, were selected to build the 
Table I. Differentially expressed IncRNAs in competing endogenous RNA network of lung adenocarcinoma.

\begin{tabular}{|c|c|c|c|c|c|c|c|}
\hline \multicolumn{4}{|c|}{ endogenous RNA network of lung adenocarcinoma. } & \\
\hline & \multirow{2}{*}{$\log \mathrm{FC}$} & \multirow{2}{*}{ P-value } & & lncRNA & $\log F C$ & P-value & FDR \\
\hline lncRNA & & & FDR & & 316 & $538 \times 10^{-10}$ & \\
\hline DSCAM-AS1 & 8.00 & $4.74 \times 10^{-12}$ & $2.80 \times 10^{-11}$ & STEAP2-AS1 & 3.14 & $2.57 \times 10^{-19}$ & $3.58 \times 10^{-18}$ \\
\hline AL160271.1 & 6.91 & $5.96 \times 10^{-10}$ & $2.64 \times 10^{-9}$ & LINC00470 & 3.08 & $2.05 \times 10^{-8}$ & $7.33 \times 10^{-8}$ \\
\hline HOTAIR & 6.77 & $8.85 \times 10^{-20}$ & $1.28 \times 10^{-18}$ & C20orf197 & 3.04 & $7.90 \times 10^{-19}$ & $1.03 \times 10^{-17}$ \\
\hline AC061975.6 & 6.53 & $5.32 \times 10^{-21}$ & $8.45 \times 10^{-20}$ & GRM7-AS3 & 3.03 & $6.73 \times 10^{-7}$ & $1.96 \times 10^{-6}$ \\
\hline CLDN10-AS1 & 6.494 & $2.04 \times 10^{-30}$ & $6.67 \times 10^{-29}$ & LSAMP-AS1 & 3.02 & $6.99 \times 10^{-8}$ & $2.33 \times 10^{-7}$ \\
\hline POU6F2-AS2 & 6.18 & $1.27 \times 10^{-14}$ & $1.02 \times 10^{-13}$ & AL354707.1 & 2.98 & $2.42 \times 10^{-29}$ & $7.35 \times 10^{-28}$ \\
\hline RMRP & 5.91 & $4.63 \times 10^{-8}$ & $1.58 \times 10^{-7}$ & FNDC1-IT1 & 2.96 & $2.65 \times 10^{-14}$ & $2.06 \times 10^{-13}$ \\
\hline NOVA1-AS1 & 5.84 & $1.20 \times 10^{-16}$ & $1.23 \times 10^{-15}$ & C2orf48 & 2.94 & $1.70 \times 10^{-28}$ & $4.95 \times 10^{-27}$ \\
\hline MUC2 & 5.81 & $4.96 \times 10^{-14}$ & $3.74 \times 10^{-13}$ & LINC00488 & 2.94 & $4.10 \times 10^{-5}$ & $9.08 \times 10^{-5}$ \\
\hline LINC00392 & 5.80 & $1.09 \times 10^{-8}$ & $4.04 \times 10^{-8}$ & CACNA1C-IT3 & 2.91 & $2.60 \times 10^{-5}$ & $5.93 \times 10^{-5}$ \\
\hline AC020907.1 & 5.79 & $9.50 \times 10^{-46}$ & $7.61 \times 10^{-44}$ & CHODL-AS1 & 2.90 & $3.14 \times 10^{-7}$ & $9.61 \times 10^{-7}$ \\
\hline ERVMER61-1 & 5.70 & $2.43 \times 10^{-10}$ & $1.13 \times 10^{-9}$ & LINC00051 & 2.90 & $1.61 \times 10^{-6}$ & $4.42 \times 10^{-6}$ \\
\hline UCA1 & 5.69 & $6.01 \times 10^{-21}$ & $9.50 \times 10^{-20}$ & AP002478.1 & 2.87 & $1.54 \times 10^{-9}$ & $6.49 \times 10^{-9}$ \\
\hline LINC00491 & 5.60 & $3.86 \times 10^{-15}$ & $3.34 \times 10^{-14}$ & AC112721.1 & 2.85 & $1.18 \times 10^{-14}$ & $9.56 \times 10^{-14}$ \\
\hline LINC00501 & 5.28 & $1.00 \times 10^{-17}$ & $1.15 \times 10^{-16}$ & LINC00337 & 2.85 & $9.70 \times 10^{-22}$ & $1.64 \times 10^{-20}$ \\
\hline LINC00221 & 5.19 & $6.98 \times 10^{-10}$ & $3.06 \times 10^{-9}$ & AP000553.1 & 2.82 & $2.69 \times 10^{-28}$ & $7.68 \times 10^{-27}$ \\
\hline AL513123.1 & 5.17 & $1.40 \times 10^{-15}$ & $1.29 \times 10^{-14}$ & TDRG1 & 2.77 & $6.14 \times 10^{-6}$ & $1.55 \times 10^{-5}$ \\
\hline NAALADL2-AS2 & 5.07 & $1.22 \times 10^{-16}$ & $1.25 \times 10^{-15}$ & E2F3-IT1 & 2.76 & $2.17 \times 10^{-6}$ & $5.87 \times 10^{-6}$ \\
\hline MIR137HG & 4.97 & $5.03 \times 10^{-15}$ & $4.30 \times 10^{-14}$ & AL021395.1 & 2.70 & $8.27 \times 10^{-6}$ & $1.75 \times 10^{-4}$ \\
\hline LINC00393 & 4.80 & $3.61 \times 10^{-15}$ & $1.43 \times 10^{-8}$ & PVT1 & 2.66 & $3.20 \times 10^{-49}$ & $3.03 \times 10^{-47}$ \\
\hline ERVH48-1 & 4.74 & $4.28 \times 10^{-16}$ & $4.12 \times 10^{-15}$ & TBL1XR1-AS1 & 2.66 & $1.99 \times 10^{-9}$ & $8.25 \times 10^{-9}$ \\
\hline AL356133.2 & 4.72 & $1.42 \times 10^{-9}$ & $6.02 \times 10^{-9}$ & HNF1A-AS1 & 2.65 & $1.65 \times 10^{-10}$ & $7.91 \times 10^{-10}$ \\
\hline LINC00518 & 4.48 & $9.96 \times 10^{-14}$ & $7.17 \times 10^{-13}$ & AL139002.1 & 2.65 & $6.36 \times 10^{-4}$ & $1.16 \times 10^{-3}$ \\
\hline DLX6-AS1 & 4.44 & $3.14 \times 10^{-16}$ & $3.07 \times 10^{-15}$ & LINC00319 & 2.62 & $1.46 \times 10^{-9}$ & $6.19 \times 10^{-9}$ \\
\hline LINC00460 & 4.43 & $9.38 \times 10^{-19}$ & $1.22 \times 10^{-17}$ & DPYD-AS2 & 2.57 & $2.80 \times 10^{-5}$ & $6.35 \times 10^{-5}$ \\
\hline LINC00355 & 4.36 & $4.32 \times 10^{-10}$ & $1.95 \times 10^{-9}$ & DSCR10 & 2.54 & $5.21 \times 10^{-5}$ & $1.14 \times 10^{-4}$ \\
\hline LINC00466 & 4.29 & $1.57 \times 10^{-16}$ & $1.58 \times 10^{-15}$ & IGF2-AS & 2.54 & $2.15 \times 10^{-6}$ & $5.82 \times 10^{-6}$ \\
\hline LINC00483 & 4.25 & $7.95 \times 10^{-13}$ & $5.15 \times 10^{-12}$ & LINC00440 & 2.48 & $9.96 \times 10^{-5}$ & $2.08 \times 10^{-4}$ \\
\hline POU6F2-AS1 & 4.16 & $2.36 \times 10^{-12}$ & $1.44 \times 10^{-11}$ & LPP-AS1 & 2.45 & $2.82 \times 10^{-4}$ & $5.46 \times 10^{-4}$ \\
\hline LINC00461 & 4.04 & $7.80 \times 10^{-24}$ & $1.59 \times 10^{-22}$ & VCAN-AS1 & 2.45 & $1.41 \times 10^{-8}$ & $5.15 \times 10^{-8}$ \\
\hline AC087269.1 & 3.81 & $9.38 \times 10^{-22}$ & $1.59 \times 10^{-20}$ & LINC00519 & 2.45 & $7.77 \times 10^{-16}$ & $7.32 \times 10^{-15}$ \\
\hline AC084262.1 & 3.74 & $1.64 \times 10^{-18}$ & $2.07 \times 10^{-17}$ & AL353803.1 & 2.41 & $1.09 \times 10^{-8}$ & $4.04 \times 10^{-8}$ \\
\hline AC010145.1 & 3.73 & $1.07 \times 10^{-5}$ & $2.58 \times 10^{-5}$ & IL20RB-AS1 & 2.40 & $8.01 \times 10^{-7}$ & $2.31 \times 10^{-6}$ \\
\hline LINC00473 & 3.65 & $6.95 \times 10^{-8}$ & $2.32 \times 10^{-7}$ & ARHGEF3-AS1 & 2.39 & $1.13 \times 10^{-4}$ & $2.34 \times 10^{-4}$ \\
\hline MYCNOS & 3.62 & $9.13 \times 10^{-12}$ & $5.17 \times 10^{-11}$ & CHL1-AS1 & 2.38 & $1.43 \times 10^{-10}$ & $6.93 \times 10^{-10}$ \\
\hline LINC00160 & 3.58 & $7.19 \times 10^{-21}$ & $1.13 \times 10^{-19}$ & ATG10-AS1 & 2.37 & $6.38 \times 10^{-4}$ & $1.17 \times 10^{-3}$ \\
\hline HOTTIP & 3.56 & $1.13 \times 10^{-7}$ & $3.65 \times 10^{-7}$ & EGOT & 2.33 & $4.72 \times 10^{-15}$ & $4.05 \times 10^{-14}$ \\
\hline AC080129.1 & 3.48 & $1.78 \times 10^{-9}$ & $7.43 \times 10^{-9}$ & C11orf44 & 2.33 & $2.95 \times 10^{-7}$ & $9.05 \times 10^{-7}$ \\
\hline AC006372.1 & 3.43 & $2.63 \times 10^{-7}$ & $8.13 \times 10^{-7}$ & SOX21-AS1 & 2.29 & $1.91 \times 10^{-10}$ & $9.02 \times 10^{-10}$ \\
\hline LINC00525 & 3.42 & $1.30 \times 10^{-23}$ & $2.55 \times 10^{-22}$ & GRM5-AS1 & 2.27 & $4.77 \times 10^{-9}$ & $1.86 \times 10^{-8}$ \\
\hline LINC00524 & 3.41 & $1.04 \times 10^{-10}$ & $5.14 \times 10^{-10}$ & U52111.1 & 2.26 & $7.60 \times 10^{-26}$ & $1.82 \times 10^{-24}$ \\
\hline WASIR2 & 3.40 & $2.06 \times 10^{-35}$ & $9.20 \times 10^{-34}$ & AC007731.1 & 2.25 & $5.46 \times 10^{-5}$ & $1.19 \times 10^{-4}$ \\
\hline H19 & 3.35 & $3.20 \times 10^{-11}$ & $1.68 \times 10^{-10}$ & AC012640.1 & 2.23 & $6.87 \times 10^{-17}$ & $7.26 \times 10^{-16}$ \\
\hline AC022148.1 & 3.34 & $2.47 \times 10^{-13}$ & $1.71 \times 10^{-12}$ & FOXP1-IT1 & 2.23 & $4.71 \times 10^{-7}$ & $1.40 \times 10^{-6}$ \\
\hline LINC00200 & 3.33 & $5.05 \times 10^{-5}$ & $1.10 \times 10^{-4}$ & AL117190.1 & 2.22 & $5.40 \times 10^{-8}$ & $1.83 \times 10^{-7}$ \\
\hline KIF25-AS1 & 3.32 & $1.98 \times 10^{-10}$ & $9.34 \times 10^{-10}$ & C1orf220 & 2.19 & $7.28 \times 10^{-40}$ & $4.06 \times 10^{-38}$ \\
\hline LINC00536 & 3.30 & $4.92 \times 10^{-11}$ & $2.51 \times 10^{-10}$ & AC092535.1 & 2.18 & $1.18 \times 10^{-12}$ & $7.50 \times 10^{-12}$ \\
\hline LINC00308 & 3.21 & $1.15 \times 10^{-7}$ & $3.74 \times 10^{-7}$ & LINC00485 & 2.15 & $1.02 \times 10^{-4}$ & $2.12 \times 10^{-4}$ \\
\hline FER1L6-AS1 & 3.21 & $4.93 \times 10^{-5}$ & $1.08 \times 10^{-4}$ & LINC00330 & 2.14 & $6.66 \times 10^{-9}$ & $2.55 \times 10^{-8}$ \\
\hline SAMSN1-AS1 & 3.18 & $1.87 \times 10^{-11}$ & $1.02 \times 10^{-10}$ & AL391152.1 & 2.14 & $5.84 \times 10^{-8}$ & $1.97 \times 10^{-7}$ \\
\hline AC026320.1 & 3.16 & $2.03 \times 10^{-6}$ & $5.53 \times 10^{-6}$ & ZBTB20-AS3 & 2.10 & $3.01 \times 10^{-3}$ & $4.95 \times 10^{-3}$ \\
\hline
\end{tabular}

Table I. Continued. 
Table I. Continued.

\begin{tabular}{llll}
\hline lncRNA & logFC & P-value & FDR \\
\hline SYNPR-AS1 & 2.08 & $2.06 \times 10^{-13}$ & $1.44 \times 10^{-12}$ \\
AL139385.1 & 2.07 & $4.99 \times 10^{-13}$ & $3.31 \times 10^{-12}$ \\
AC110921.1 & 2.07 & $1.13 \times 10^{-4}$ & $2.33 \times 10^{-4}$ \\
MEG3 & 2.06 & $1.96 \times 10^{-10}$ & $9.25 \times 10^{-10}$ \\
HECW1-IT1 & 2.04 & $9.40 \times 10^{-4}$ & $1.67 \times 10^{-3}$ \\
ANO1-AS2 & 2.03 & $2.62 \times 10^{-5}$ & $5.96 \times 10^{-5}$ \\
ARHGAP26-AS1 & 2.02 & $1.51 \times 10^{-7}$ & $4.80 \times 10^{-7}$ \\
LINC00184 & 2.01 & $7.45 \times 10^{-11}$ & $3.74 \times 10^{-10}$ \\
AL365356.1 & 2.01 & $1.74 \times 10^{-7}$ & $5.48 \times 10^{-7}$ \\
C10orf91 & 2.01 & $2.52 \times 10^{-13}$ & $1.74 \times 10^{-12}$ \\
AC016773.1 & 2.01 & $3.08 \times 10^{-31}$ & $1.06 \times 10^{-29}$ \\
AP000525.1 & 2.00 & $6.49 \times 10^{-12}$ & $3.75 \times 10^{-11}$ \\
HHATL-AS1 & -2.00 & $6.24 \times 10^{-14}$ & $4.62 \times 10^{-13}$ \\
AGAP11 & -2.07 & $1.06 \times 10^{-35}$ & $4.79 \times 10^{-34}$ \\
RMST & -2.10 & $2.26 \times 10^{-17}$ & $2.51 \times 10^{-16}$ \\
AC025431.1 & -2.12 & $2.42 \times 10^{-15}$ & $2.15 \times 10^{-14}$ \\
C5orf64 & -2.12 & $1.67 \times 10^{-40}$ & $9.91 \times 10^{-39}$ \\
TTTY16 & -2.14 & $3.44 \times 10^{-9}$ & $1.37 \times 10^{-8}$ \\
LINC00472 & -2.17 & $3.92 \times 10^{-44}$ & $2.89 \times 10^{-42}$ \\
AC004832.1 & -2.20 & $8.05 \times 10^{-22}$ & $1.37 \times 10^{-20}$ \\
MED4-AS1 & -2.28 & $6.93 \times 10^{-63}$ & $1.04 \times 10^{-60}$ \\
SRGAP3-AS2 & -2.30 & $2.17 \times 10^{-14}$ & $1.70 \times 10^{-13}$ \\
LINC00211 & -2.31 & $2.04 \times 10^{-36}$ & $9.54 \times 10^{-35}$ \\
MYO16-AS1 & -2.36 & $5.18 \times 10^{-17}$ & $5.57 \times 10^{-16}$ \\
AP003064.2 & -2.46 & $9.03 \times 10^{-27}$ & $2.33 \times 10^{-25}$ \\
ADAMTS9-AS1 & -2.77 & $4.41 \times 10^{-75}$ & $1.20 \times 10^{-72}$ \\
NAV2-AS2 & -2.78 & $8.94 \times 10^{-44}$ & $6.27 \times 10^{-42}$ \\
AC105206.1 & -2.96 & $5.33 \times 10^{-27}$ & $1.41 \times 10^{-25}$ \\
AL109754.1 & -2.96 & $2.39 \times 10^{-43}$ & $1.63 \times 10^{-41}$ \\
AP000438.1 & -3.01 & $2.80 \times 10^{-72}$ & $6.44 \times 10^{-70}$ \\
\hline
\end{tabular}

lncRNA, long non-coding RNA; FC, fold-change; FDR, false discovery rate.

ceRNA network (Table I). Next, the miRDB, miRTarBase and TargetScan online tools were used to predict mRNAs targeted by miRNAs. The targeting associations between 21 miRNAs (17 upregulated and 4 downregulated miRNAs; Table II) and 34 mRNAs (25 upregulated and 9 downregulated mRNAs; Table III) were obtained and selected for ceRNA network construction.

Subsequently, the interactions between 21 miRNAs and 134 lncRNAs were assessed, as well as those between 11 miRNAs and 34 mRNAs (data not shown). Based on these targeting associations, the lncRNA-miRNA-mRNA ceRNA network was constructed using Cytoscape version 3.5.1. According to the expression levels of differentially expressed mRNAs, lncRNAs and miRNAs, two ceRNA networks, namely overexpression and underexpression networks, were constructed (Fig. 2).
Table II. Differentially expressed miRNAs in the competing endogenous RNA network of lung adenocarcinoma.

\begin{tabular}{llll}
\hline miRNA & logFC & P-value & FDR \\
\hline hsa-mir-372 & 7.08 & $5.63 \times 10^{-9}$ & $2.36 \times 10^{-8}$ \\
hsa-mir-122 & 5.91 & $1.51 \times 10^{-6}$ & $4.54 \times 10^{-6}$ \\
hsa-mir-373 & 5.50 & $3.48 \times 10^{-6}$ & $9.72 \times 10^{-6}$ \\
hsa-mir-210 & 5.07 & $1.11 \times 10^{-58}$ & $6.33 \times 10^{-57}$ \\
hsa-mir-137 & 4.42 & $3.31 \times 10^{-13}$ & $2.06 \times 10^{-12}$ \\
hsa-mir-31 & 4.37 & $2.23 \times 10^{-17}$ & $1.81 \times 10^{-16}$ \\
hsa-mir-301b & 3.60 & $1.99 \times 10^{-22}$ & $2.06 \times 10^{-21}$ \\
hsa-mir-215 & 2.95 & $3.54 \times 10^{-9}$ & $1.52 \times 10^{-8}$ \\
hsa-mir-192 & 2.81 & $5.71 \times 10^{-11}$ & $2.98 \times 10^{-10}$ \\
hsa-mir-205 & 2.73 & $6.86 \times 10^{-9}$ & $2.78 \times 10^{-8}$ \\
hsa-mir-96 & 2.72 & $1.42 \times 10^{-40}$ & $3.74 \times 10^{-39}$ \\
hsa-mir-489 & 2.55 & $1.06 \times 10^{-7}$ & $3.76 \times 10^{-7}$ \\
hsa-mir-503 & 2.48 & $1.45 \times 10^{-21}$ & $1.43 \times 10^{-20}$ \\
hsa-mir-216b & 2.44 & $1.53 \times 10^{-5}$ & $4.05 \times 10^{-5}$ \\
hsa-mir-187 & 2.38 & $8.58 \times 10^{-11}$ & $4.38 \times 10^{-10}$ \\
hsa-mir-183 & 2.36 & $4.29 \times 10^{-33}$ & $9.46 \times 10^{-32}$ \\
hsa-mir-182 & 2.05 & $1.31 \times 10^{-29}$ & $2.25 \times 10^{-28}$ \\
hsa-mir-195 & -2.27 & $4.49 \times 10^{-90}$ & $7.68 \times 10^{-88}$ \\
hsa-mir-143 & -2.75 & $4.79 \times 10^{-88}$ & $6.56 \times 10^{-86}$ \\
hsa-mir-184 & -2.91 & $4.74 \times 10^{-28}$ & $7.20 \times 10^{-27}$ \\
hsa-mir-144 & -3.42 & $4.43 \times 10^{-98}$ & $1.01 \times 10^{-95}$ \\
\hline
\end{tabular}

FC, fold-change; FDR, false discovery rate; miRNA/miR, microRNA.

Functional enrichment analysis. To further predict putative disease prognosis-related biomarkers and the biological processes and pathways to which they belong, functional enrichment analysis of IncRNAs in the ceRNA networks was performed for GO terms and KEGG pathways. Differentially expressed mRNAs targeted by lncRNAs in the ceRNA networks were analyzed using the DAVID database. In total, 2,507 differentially expressed mRNAs were identified, including 1,977 upregulated and 527 downregulated mRNAs from LUAC tissues, when compared with adjacent normal samples based on $\mathrm{P}$-values of $<0.01$ and $\log \mathrm{FCl}>2$. Functional annotation indicated that upregulated mRNAs were involved in $23 \mathrm{GO}$ terms, most significantly in 'DNA replication', 'G1/S transition of the mitotic cell cycle' and 'cell cycle regulation'. These genes were mainly enriched in 'cell cycle' and 'p53 signaling pathways'. By contrast, downregulated genes were found to be associated with GO terms of 'BMP signaling pathway', 'integral component of membrane', 'extracellular region' and 'perinuclear region of cytoplasm' (Table IV).

Determination andanalysis of predictive prognostic signature. Since the selected differentially expressed mRNAs, IncRNAs and miRNAs in the ceRNA network exhibited distinct expression patterns in patients with LUAC, these coding and non-coding ceRNAs were analyzed using Kaplan-Meier and log-rank test methods to predict the prognosis of such patients. A total of 8 differentially expressed lncRNA ceRNAs were identified, including AP000525.1, AP002478.1, LINC00518, 
A

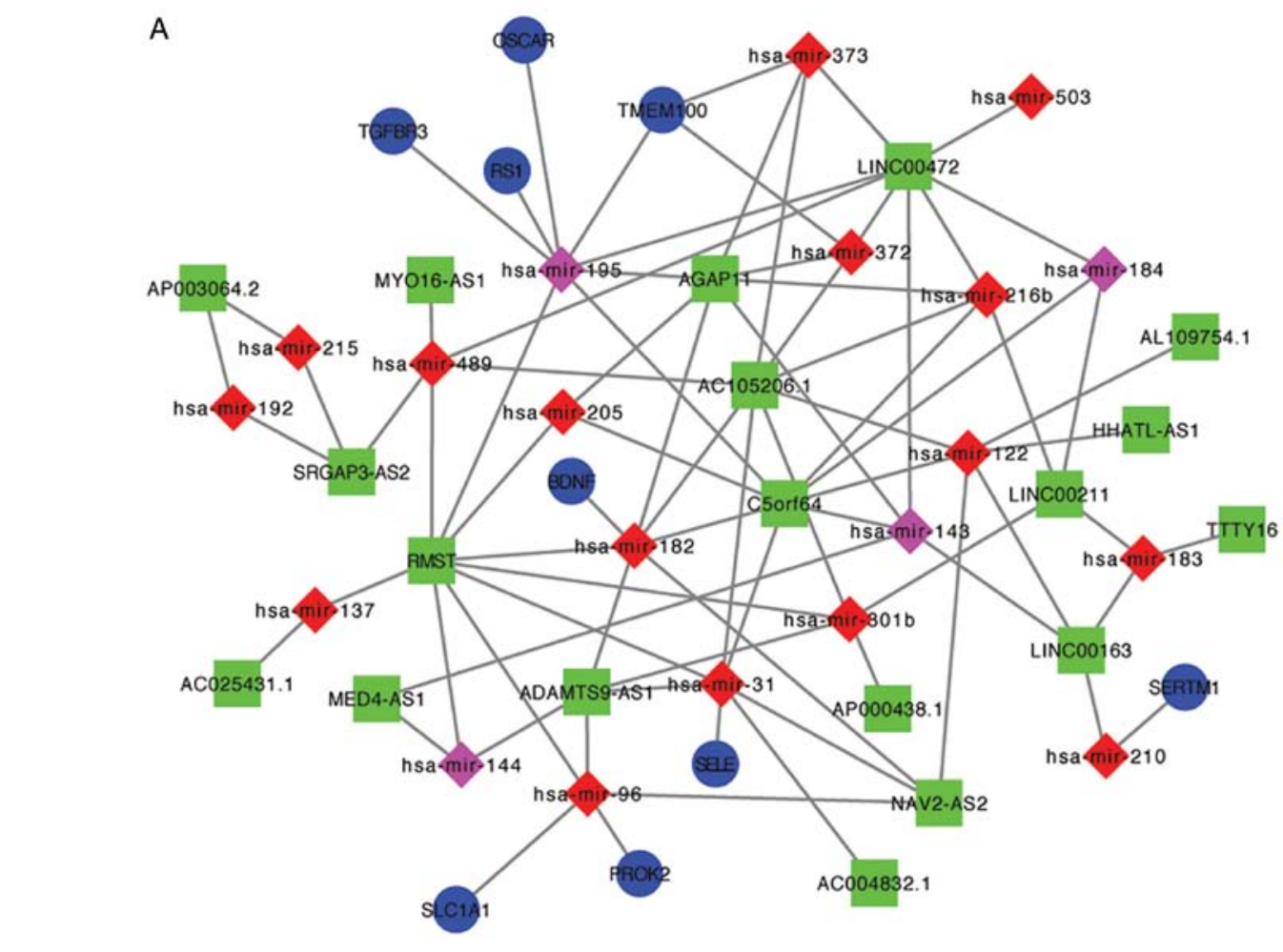

B

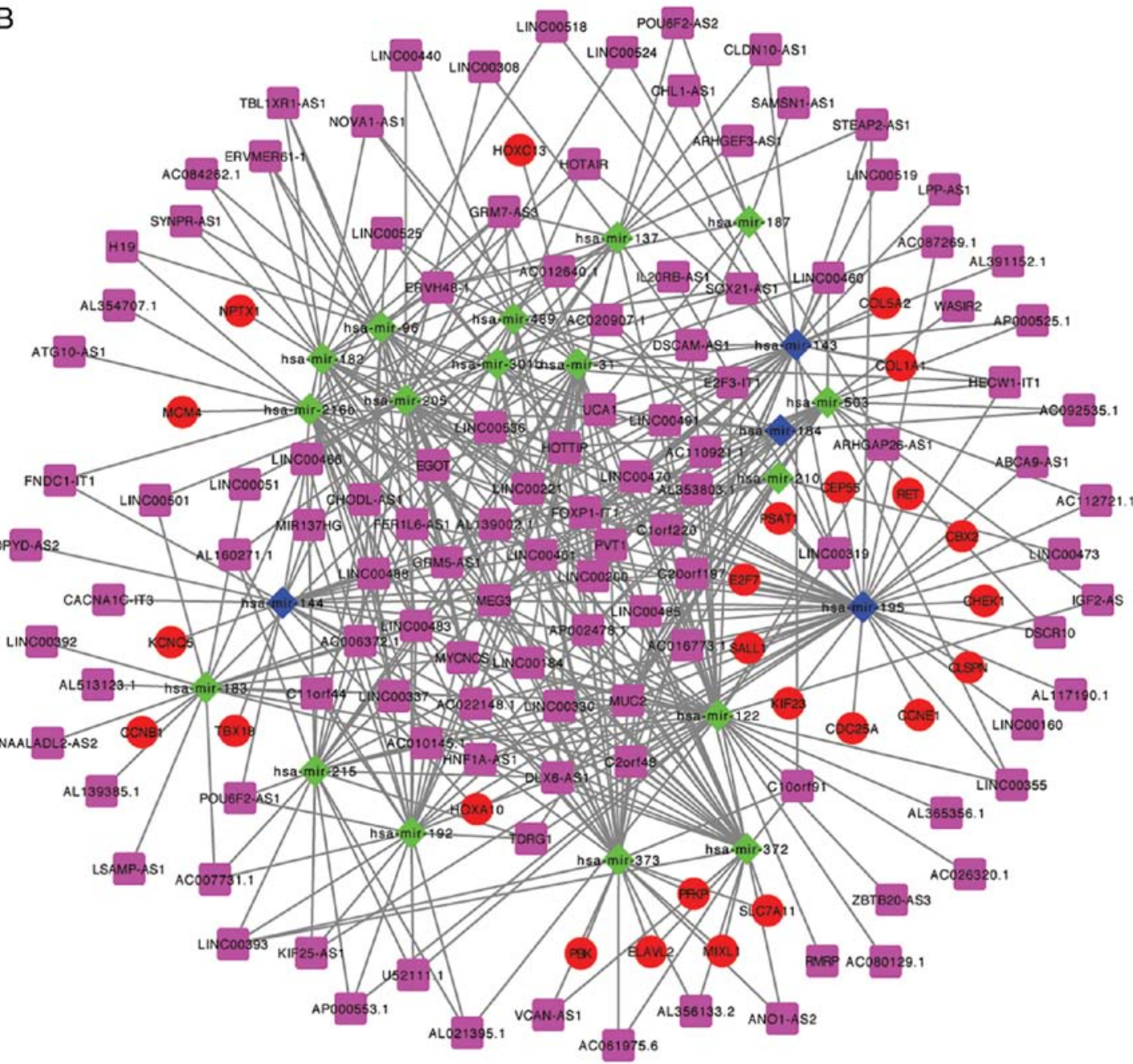

Figure 2. IncRNA-mRNA-miRNA ceRNA network of (A) underexpressed and (B) overexpressed lncRNAs, mRNAs and miRNAs. In network A, blue circles represent underexpressed mRNAs, green rectangles represent underexpressed lncRNAs, red diamonds represent overexpressed miRNAs and pink diamonds represent underexpressed miRNAs. In network B, red circles represent overexpressed mRNAs, pink rectangles represent overexpressed lncRNAs, green diamonds represent overexpressed miRNAs and blue diamonds represent underexpressed miRNAs. IncRNA, long non-coding RNA; miRNA, microRNA. 
Table III. Differentially expressed mRNA in the competing endogenous RNA network of lung adenocarcinoma.

\begin{tabular}{|c|c|c|c|}
\hline mRNA & $\log F C$ & P-value & FDR \\
\hline HOXC13 & 6.96 & $1.73 \times 10^{-22}$ & $1.34 \times 10^{-21}$ \\
\hline SALL1 & 5.81 & $6.30 \times 10^{-17}$ & $3.21 \times 10^{-16}$ \\
\hline HOXA10 & 4.22 & $4.90 \times 10^{-22}$ & $3.67 \times 10^{-21}$ \\
\hline NPTX1 & 3.76 & $4.78 \times 10^{-16}$ & $2.26 \times 10^{-15}$ \\
\hline PSAT1 & 3.52 & $3.76 \times 10^{-48}$ & $1.06 \times 10^{-46}$ \\
\hline ELAVL2 & 3.47 & $1.09 \times 10^{-15}$ & $5.03 \times 10^{-15}$ \\
\hline PBK & 3.41 & $3.11 \times 10^{-43}$ & $7.08 \times 10^{-42}$ \\
\hline CCNE1 & 3.31 & $1.55 \times 10^{-42}$ & $3.43 \times 10^{-41}$ \\
\hline CEP55 & 3.28 & $1.30 \times 10^{-55}$ & $5.03 \times 10^{-54}$ \\
\hline SLC7A11 & 3.09 & $2.38 \times 10^{-23}$ & $1.96 \times 10^{-22}$ \\
\hline CCNB1 & 3.00 & $8.18 \times 10^{-55}$ & $3.01 \times 10^{-53}$ \\
\hline RET & 2.97 & $1.80 \times 10^{-13}$ & $6.93 \times 10^{-13}$ \\
\hline COL1A1 & 2.91 & $9.49 \times 10^{-32}$ & $1.27 \times 10^{-30}$ \\
\hline E2F7 & 2.76 & $3.91 \times 10^{-31}$ & $5.04 \times 10^{-30}$ \\
\hline CLSPN & 2.75 & $4.81 \times 10^{-40}$ & $9.63 \times 10^{-39}$ \\
\hline TBX18 & 2.69 & $2.39 \times 10^{-10}$ & $7.10 \times 10^{-10}$ \\
\hline KCNQ5 & 2.65 & $4.31 \times 10^{-20}$ & $2.80 \times 10^{-19}$ \\
\hline KIF23 & 2.61 & $6.02 \times 10^{-43}$ & $1.35 \times 10^{-41}$ \\
\hline $\mathrm{CBX} 2$ & 2.52 & $1.99 \times 10^{-25}$ & $1.86 \times 10^{-24}$ \\
\hline $\mathrm{CDC} 25 \mathrm{~A}$ & 2.37 & $1.98 \times 10^{-37}$ & $3.51 \times 10^{-36}$ \\
\hline CHEK1 & 2.26 & $1.69 \times 10^{-43}$ & $3.89 \times 10^{-42}$ \\
\hline MCM4 & 2.24 & $4.41 \times 10^{-47}$ & $1.17 \times 10^{-45}$ \\
\hline COL5A2 & 2.20 & $5.53 \times 10^{-28}$ & $6.09 \times 10^{-27}$ \\
\hline PFKP & 2.17 & $2.44 \times 10^{-33}$ & $3.57 \times 10^{-32}$ \\
\hline MIXL1 & 2.00 & $5.19 \times 10^{-17}$ & $2.66 \times 10^{-16}$ \\
\hline PROK2 & -2.02 & $1.49 \times 10^{-24}$ & $1.32 \times 10^{-23}$ \\
\hline SLC1A1 & -2.17 & $1.86 \times 10^{-50}$ & $5.75 \times 10^{-49}$ \\
\hline OSCAR & -2.20 & $3.65 \times 10^{-67}$ & $2.25 \times 10^{-65}$ \\
\hline BDNF & -2.31 & $2.27 \times 10^{-32}$ & $3.15 \times 10^{-31}$ \\
\hline TGFBR3 & -2.53 & $1.67 \times 10^{-87}$ & $1.77 \times 10^{-85}$ \\
\hline SELE & -2.90 & $2.85 \times 10^{-63}$ & $1.50 \times 10^{-61}$ \\
\hline RS1 & -3.70 & $1.04 \times 10^{-94}$ & $1.30 \times 10^{-92}$ \\
\hline TMEM100 & -4.31 & $1.71 \times 10^{-143}$ & $7.55 \times 10^{-141}$ \\
\hline SERTM1 & -4.74 & $3.04 \times 10^{-70}$ & $2.05 \times 10^{-68}$ \\
\hline
\end{tabular}

FC, fold-change; FDR, false discovery rate.

MED4-antisense 1 (AS1), NAV2-AS2, STEAP2-AS1, SYNPR-AS1 and urothelial cancer-associated 1, as well as 9 differentially expressed mRNA ceRNAs, including cyclin B1 $(C C N B 1)$, centrosomal protein 55 (CEP55), checkpoint kinase 1 (CHEK1), E2F transcription factor 7 (E2F7), kinesin family member 23 (KIF23), minichromosome maintenance complex component 4, PDZ binding kinase, phosphofructokinase platelet and retinoschisin $1(R S 1)$, which were associated with overall survival (Figs. 3 and 4). Subsequent to univariate Cox's proportional hazards regression model analysis for differentially expressed lncRNAs in the ceRNA networks,
19 lncRNAs were selected to have a significant prognostic value (data not shown), but $22 \mathrm{lncRNAs}$ and mRNAs from the ceRNA networks were identified by integrated univariate Cox's model analysis as aberrantly expressed lncRNAs and mRNAs (data not shown). Based on the criterion of a P-value of $<0.01$, the selected lncRNA and mRNA ceRNAs were used to build IncRNA- or IncRNA-mRNA-based prognostic signatures using a multivariate Cox's regression model. The results showed that 9 lncRNA ceRNAs were included in a lncRNA-based prognostic signature (termed LASiglnc-9), and two IncRNA and three mRNA ceRNAs were included in a lncRNA-mRNA-based prognostic signature (termed LASiglnc2-m3) (Fig. 5). The prognostic risk score for predicting overall survival was calculated as: $\exp _{1} * \beta_{1}+\exp _{2} * \beta_{2}+\ldots+\exp _{\mathrm{n}} * \beta_{\mathrm{n}}$. The median was used as the cutoff of risk score, and LUAC patients were divided into high-risk and low-risk groups based on this categorization (Fig. 5). Differentially expressed lncRNAs and mRNAs included in the two models are shown in Fig. 5, these include ABCA9-AS1, MED4-AS1, C5orf64, AP000438.1, LINC00319, LINC00518, C20orf197, LINC00460, LINC00519, CCNB1, KIF23 and E2F7. The time-dependent ROC curves analysis for LASiglnc-9 achieved an area under the curve (AUC) of 0.701 for the 5-year survival of LUAC patients (Fig. 6A) and the survival rate of the low-risk group was higher than that of high-risk group $(\mathrm{P}<0.001$; Fig. 6B). The time-dependent ROC curve analysis of LASiglnc2-m3 achieved an AUC of 0.627 (Fig. 6C) and the survival rate was similar to that of LASiglnc-9 (Fig. 6D). These results suggest that the accuracy of LASiglnc-9 is higher than that of LASiglnc2-m3 for predicting LUAC prognosis functioned as ceRNAs.

To further study the value of LASiglnc-9 for LUAC prognosis, the expression pattern of 9 lncRNAs of tumor patients in two risk groups was analyzed and presented in Fig. 7. Of these 9 lncRNAs, the expression of 5 lncRNAs (LINC00460, LINC00519, LINC00518, ABCA9-AS1 and LINC00319) was higher in the high-risk group than that in the low-risk group $(\mathrm{P}<0.001)$, while the expression of the other $4 \operatorname{lncRNAs}$ (AP000438.1, MED4-AS1, C5orf64 and C20orf197) was lower in the high-risk group than that in the low-risk group $(\mathrm{P}<0.001)$.

Independence of predictive capacity of LASiglnc-9 from clinical factors. Kaplan-Meier curve analysis for clinical factors, including age, gender, stage of pathology, and T, $\mathrm{N}$ and $\mathrm{M}$ stages, revealed that stage of pathology $(\mathrm{P}<0.001)$, T stage $\left(\mathrm{P}=9 \times 10^{-5}\right)$ and $\mathrm{N}$ stage $(\mathrm{P}<0.001)$ were associated with overall survival in LUAC patients (Fig. 8). Univariate Cox's regression model analysis showed that stage of pathology (HR, 2.82; 95\% CI, 1.94-4.09; $\mathrm{P}<0.001)$, T stage (HR, 2.49; 95\% CI, 1.55-4.00; $\mathrm{P}<0.001$ ), N stage (HR, 2.78; 95\% CI, 1.92-4.01; $\mathrm{P}<0.001)$ and risk score (HR, 0.39; 95\% CI, 0.26-0.58; $\mathrm{P}<0.001)$ were significantly associated with overall survival $(\mathrm{P}<0.001)$. However, T stage (HR, 1.46; 95\% CI, 0.85-2.50; $\mathrm{P}=0.170$ ) was not associated with overall survival in LUAC patients upon multivariate regression analysis (Table V). These results suggest that the stage of pathology, $\mathrm{N}$ stage and the risk score based on LASiglnc-9 function as independent prognostic factors. 

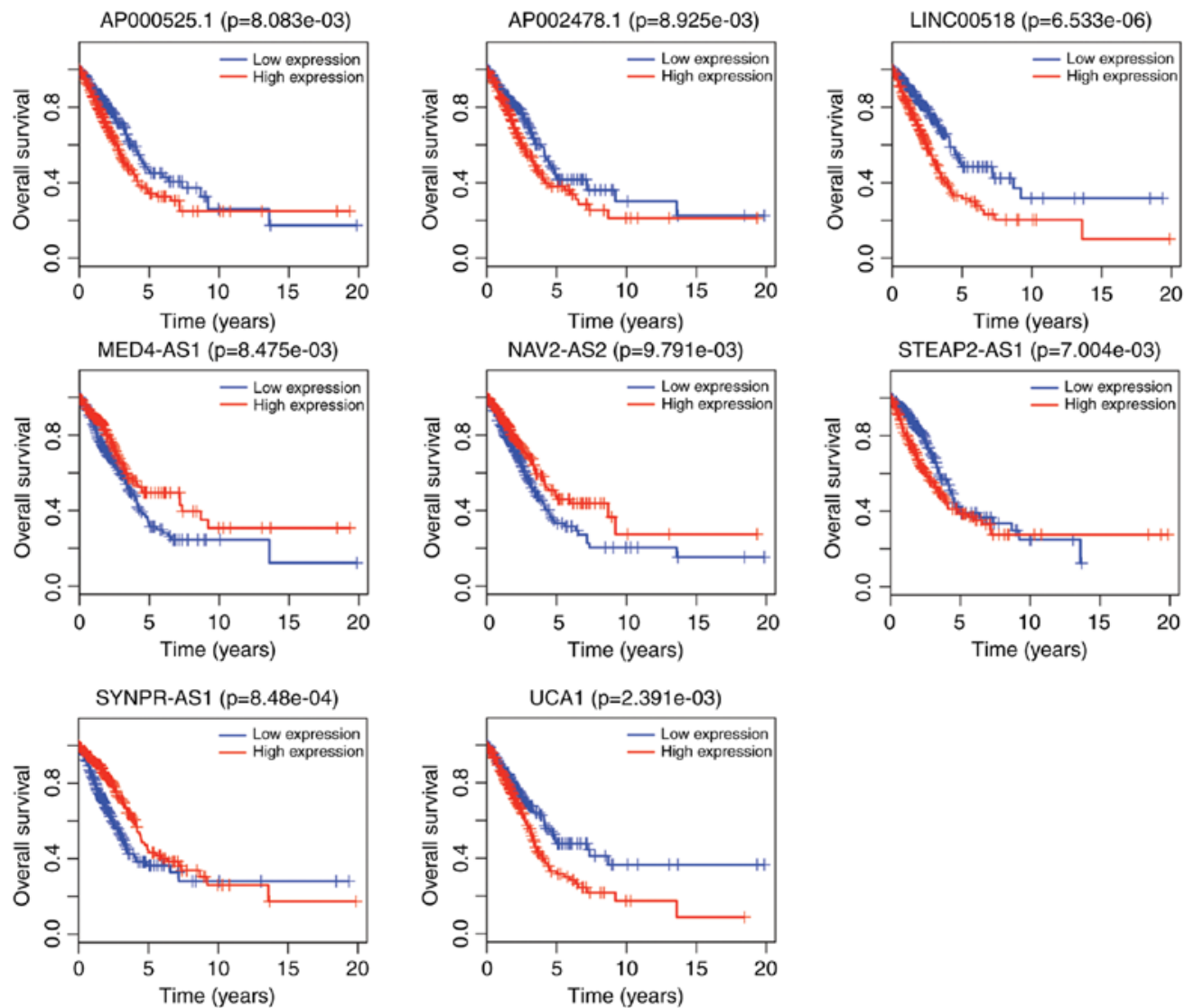

Figure 3. Kaplan-Meier survival curves for 8 lncRNAs associated with overall survival of patients with lung adenocarcinoma. Horizontal axis, overall survival time in years; vertical axis, survival function.
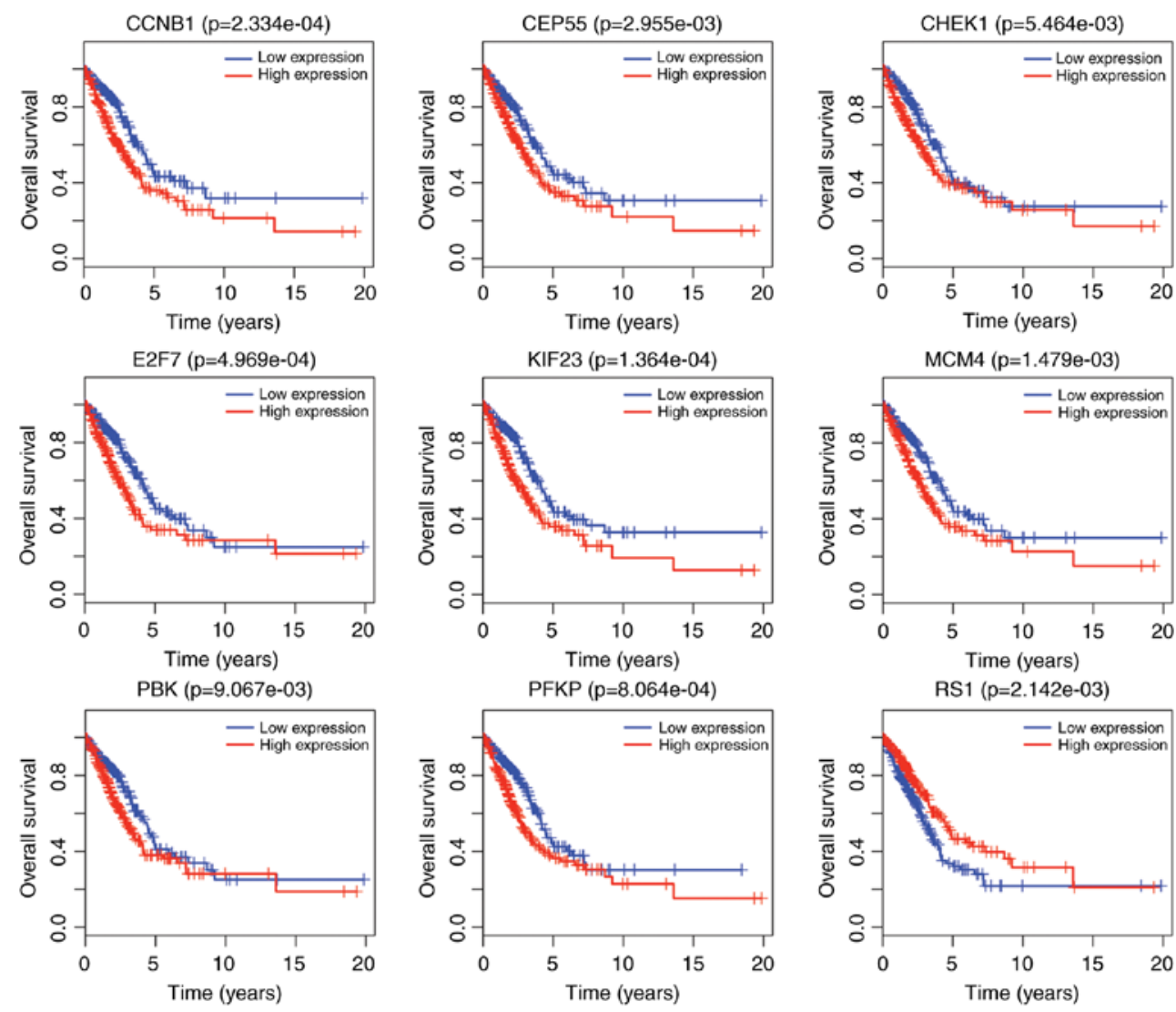

Figure 4. Kaplan-Meier survival curves for $9 \mathrm{mRNAs}$ associated with overall survival of lung adenocarcinoma. Horizontal axis, overall survival time in years; vertical axis, survival function. $C C N B 1$, cyclin B1; CEP55, centrosomal protein 55; $C H E K 1$, checkpoint kinase 1; E2F7, E2F transcription factor 7; KIF23, kinesin family member 23; MCM4, minichromosome maintenance complex component 4; PBK, PDZ binding kinase; PFKP, phosphofructokinase platelet; RS1, retinoschisin 1. 
A
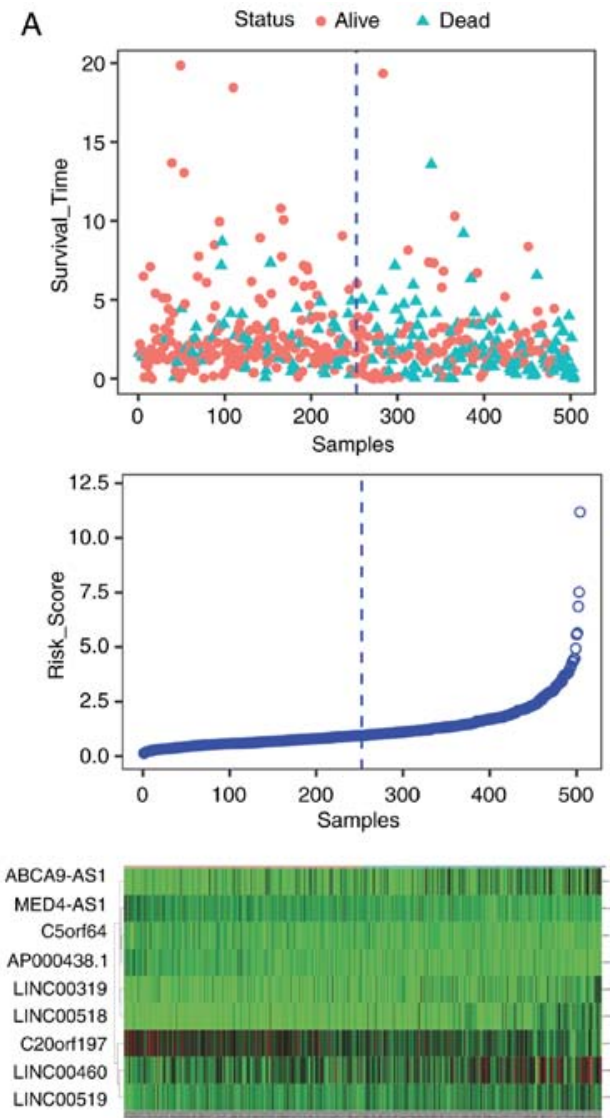

B
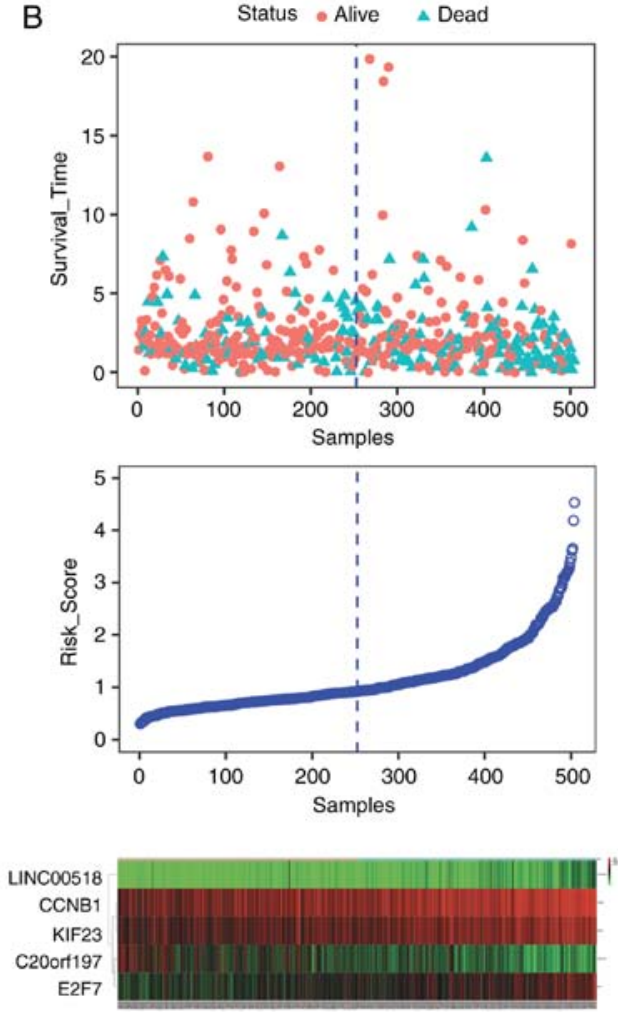

Figure 5. Risk score analysis of two prognostic signatures associated with overall survival in patients with LUAC. (A) 9-IncRNA signature LASiglnc-9 and (B) LASiglnc2-m3 signature. Survival status and duration of cases (top panels); risk score of lncRNA signature (middle panels); and heat map of LUAC-specific lncRNAs and mRNAs (bottom panels). $C C N B 1$, cyclin B1; KIF23, kinesin family member 23; E2F7, E2F transcription factor 7; LUAC, lung adenocarcinoma.

A

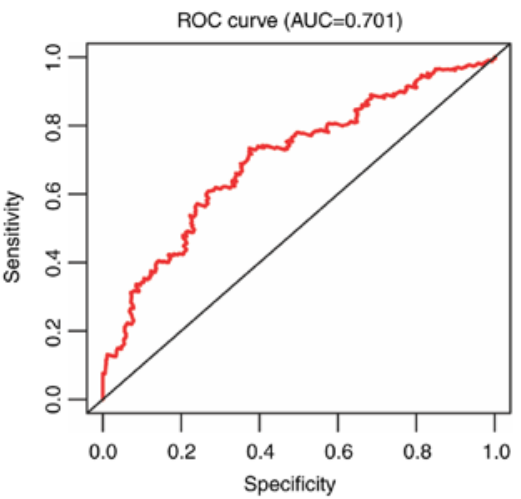

B

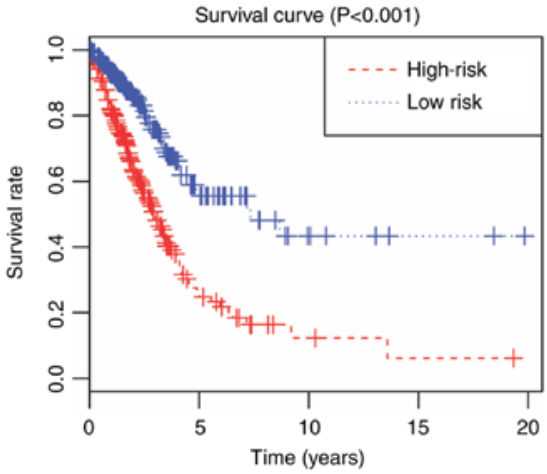

C

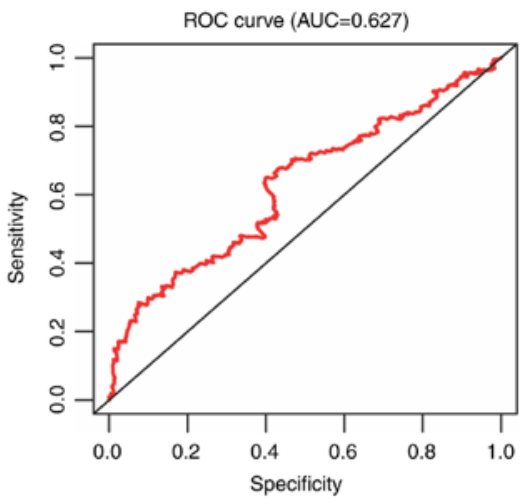

D

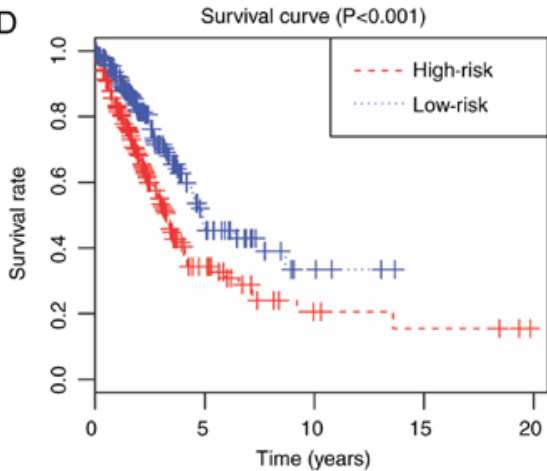

Figure 6. Two prognostic signatures, LASiglnc-9 and LASiglnc2-m3, lung adenocarcinoma outcome. (A) The ROC curve for 5-year overall survival prediction using the LASiglnc-9 signature. (B) The Kaplan-Meier curve of the risk score for the overall survival using the LASiglnc-9 signature; the log-rank test was used to compare the difference between low- and high-risk groups. (C) The ROC curve for predicting 5-year survival using the LASiglnc2-m3 signature. (D) The Kaplan-Meier curve of the risk score for the overall survival using the LASiglnc2-m3 signature. ROC, receiver operating characterstic; AUC, area under the curve. 


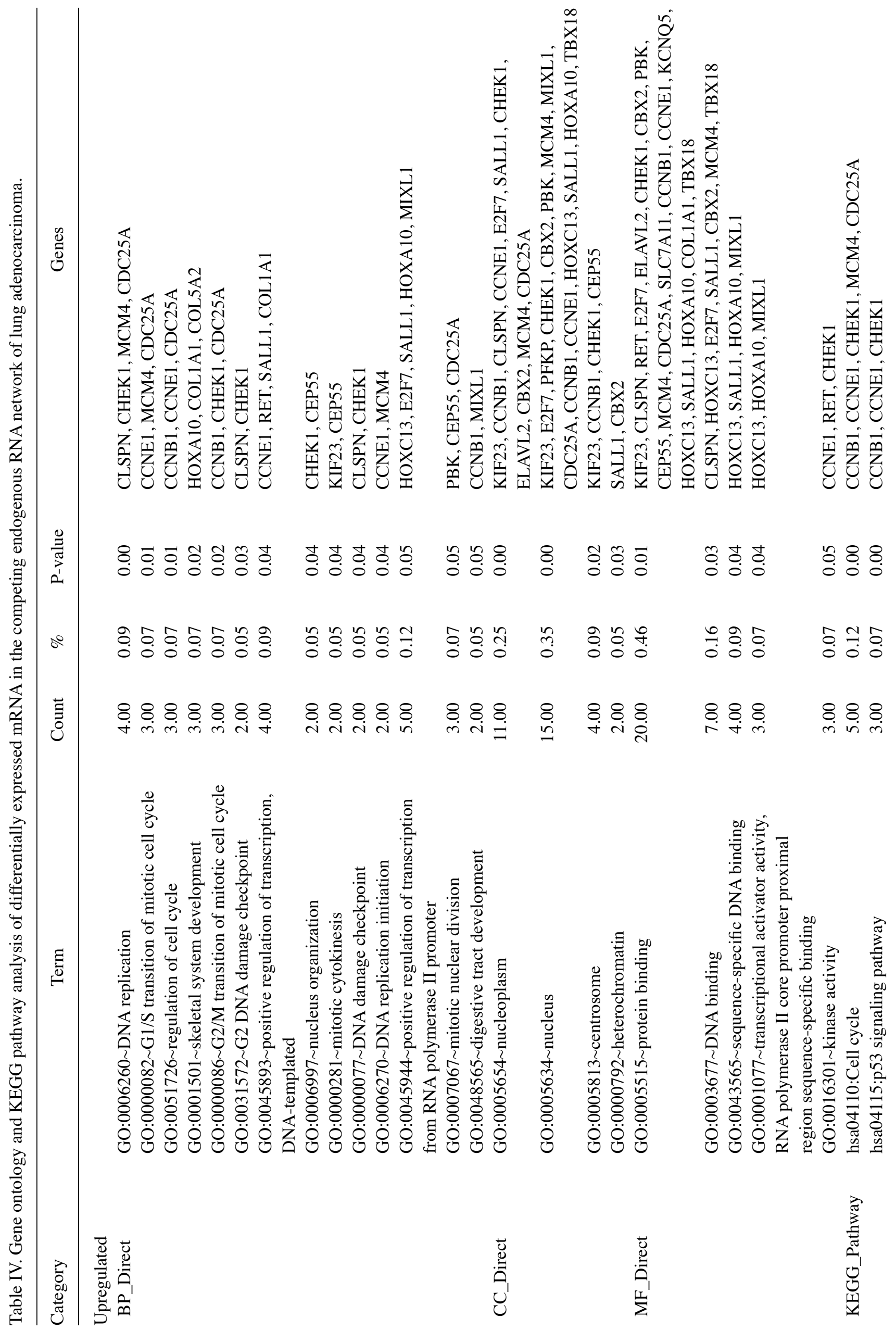




\section{Discussion}

Although clinical management of lung cancer has improved over the years through a variety of technologies that reduce patient mortality rate, an ever-increasing number of patients remain in danger of tumor recurrence or mortality (1). This is mainly due to the fact that the majority of lung cancer cases are diagnosed at advanced stages where surgical resection is not a good choice for tumor cure. Moreover, clinicopathological factors, including tumor stage, lymph node status, tumor grade and size, and lymphatic and vascular invasion appear to be associated with LUAC prognosis, but do not appear to be sufficient for predicting treatment outcomes in LUAC patients (24). A growing number of studies are focusing on microarray technology and high-throughput sequencing with the hope of identifying molecular signatures, including protein-coding genes, lncRNAs or miRNAs, that can assist in predicting survival, metastasis and the prognosis of patients $(17,25)$. Furthermore, with a greater understanding of RNA crosstalk and interaction in the scientific community, the integrated analysis of an IncRNA-associated ceRNA network is becoming more widely used to predict prognostic signatures in various cancer types, including LUAC (26). Although several lncRNAs and miRNAs have been associated with LUAC prognosis $(17,25)$, their expression patterns and prognostic values have not been thoroughly studied and they cannot be considered to be valid prognostic biomarkers at this time.

In the current study, RNA-sequencing and clinical data were retrieved from TCGA database and then analyzed and screened for differentially expressed mRNAs, IncRNAs and miRNAs between LUAC patient tissues and adjacent normal tissues. With LUAC-specific dysregulated lncRNAs, miRNAs and mRNAs, the IncRNA-mRNA-miRNA ceRNA network was constructed, which provides more insight into the detection of key RNAs associated with LUAC prognosis. Kaplan-Meier and log-rank analyses revealed 8 differentially expressed lncRNAs and 9 mRNAs associated with overall survival from exhibiting as ceRNA in patients with LUAC. Next, an lncRNA-based prognostic signature, LASiglnc-9, was constructed, which contains 9 lncRNAs, as well as an IncRNA-mRNA-based prognostic signature, LASiglnc2-m3, which contains 2 lncRNAs and 3 mRNAs based on the differentially expressed RNAs that were mapped into the ceRNA network. Of these, LASiglnc-9 showed that it may be able to more accurately predict the overall survival of patients with LUAC compared with LASiglnc2-m3. Furthermore, it was found that the predictive ability of LASiglnc-9 is certainly independent from clinicopathological factors, including stage of pathology (HR, 2.82; 95\% CI, 1.94-4.09; $\mathrm{P}<0.001$ ), T stage (HR, 2.49; 95\% CI, 1.55-4.00; $\mathrm{P}<0.001$ ), N stage (HR, 2.78; 95\% CI, 1.92-4.01; $\mathrm{P}<0.001)$ and risk score (HR, 0.39; 95\% CI, 0.26-0.58; $\mathrm{P}<0.001$ ) through Cox's regression analysis. These findings show that LASiglnc-9 may be a candidate biomarker for LUAC prognosis prediction based on mechanisms derived from the ceRNA networks.

The lncRNA ABCA9-AS1, 1 of 9 prognosis-related lncRNAs, is targeted by hsa-mir-195 in the present ceRNA network of downregulated IncRNAs and mRNAs. It is well known that hsa-mir-195 is implicated in various cancer types, 

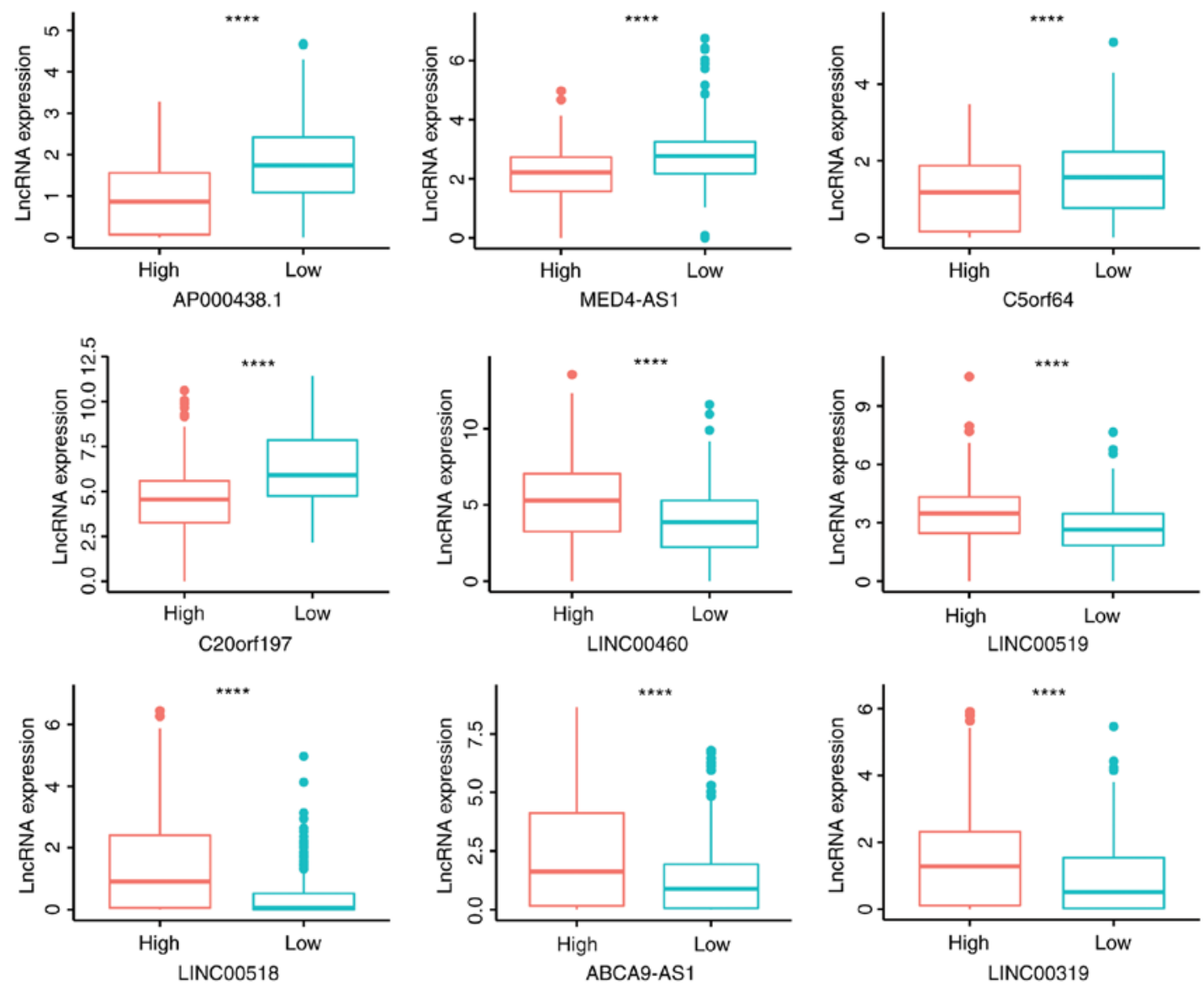

Figure 7. Expression patterns of 9 lncRNAs in high- and low-risk groups. ${ }^{* * * *} \mathrm{P}<0.001$ for high- vs. low-risk groups. lncRNA, long non-coding RNA.
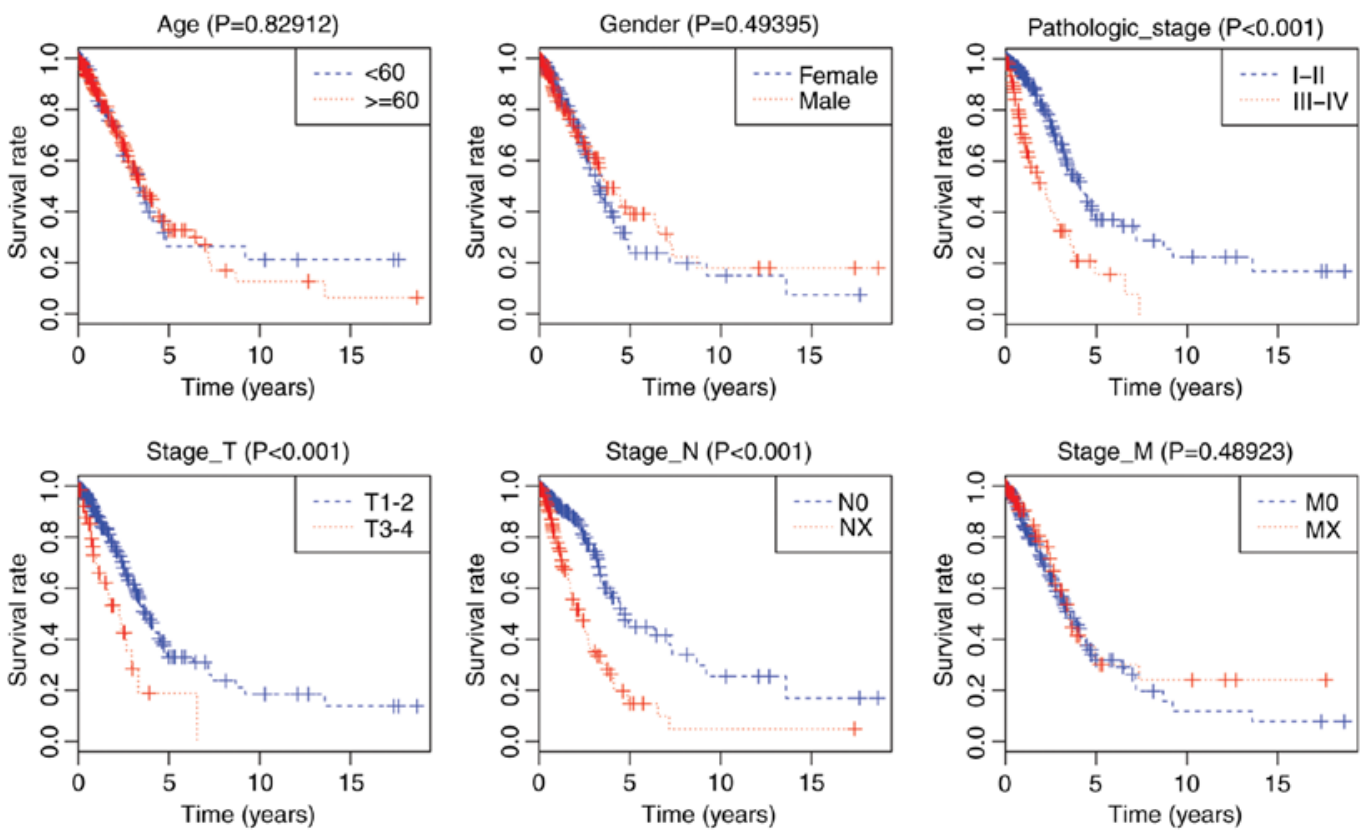

Figure 8. Prognostic value of different clinical factors for overall survival of patients with lung adenocarcinoma. Kaplan-Meier curves of six prognostic indicators.

including hepatocellular carcinoma (27), esophageal squamous cell carcinoma (28) and glioblastoma (29). Notably, a previous study demonstrated that serum mir-195 was predictive of the recurrence risk of adrenocortical cancer (30). Moreover, target prediction analysis in the present study showed that hsa-mir-195 may regulate the expression of several mRNAs in the ceRNA network, including $R S 1$, transmembrane protein 100 , osteoclast-associated immunoglobulin-like receptor, 
Table V. Predictive values of clinical features and risk score.

\begin{tabular}{|c|c|c|c|c|c|}
\hline \multirow[b]{2}{*}{ Variables } & \multirow[b]{2}{*}{ Patients, $\mathrm{n}$} & \multicolumn{2}{|c|}{ Univariate analysis } & \multicolumn{2}{|c|}{ Multivariate analysis } \\
\hline & & $\mathrm{HR}(95 \% \mathrm{CI})$ & P-value & $\mathrm{HR}(95 \% \mathrm{CI})$ & P-value \\
\hline Age $(<60 / \geq 60$ years $)$ & $131 / 351$ & $1.04(0.70-1.56)$ & 0.83 & $1.17(0.77-1.77)$ & 0.47 \\
\hline Gender (male/female) & $260 / 222$ & $0.88(0.61-1.27)$ & 0.49 & $0.82(0.57-1.20)$ & 0.31 \\
\hline Pathological stage (I-II/III-IV) & $377 / 105$ & $2.82(1.94-4.09)$ & 0.00 & $1.68(1.02-2.77)$ & 0.04 \\
\hline T stage (T1-T2/T3-T4) & $417 / 65$ & $2.49(1.55-4.00)$ & 0.00 & $1.46(0.85-2.50)$ & 0.17 \\
\hline $\mathrm{N}$ stage (N0/NX) & $312 / 170$ & $2.78(1.92-4.01)$ & 0.00 & $1.80(1.15-2.83)$ & 0.01 \\
\hline M stage (M0/MX) & $318 / 164$ & $0.87(0.58-1.30)$ & 0.49 & $0.90(0.59-1.36)$ & 0.61 \\
\hline Risk score (low/high) & $238 / 244$ & $0.39(0.26-0.58)$ & 0.00 & $0.51(0.34-0.76)$ & 0.00 \\
\hline
\end{tabular}

HR, hazard ratio; $\mathrm{CI}$, confidence interval.

transforming growth factor $\beta$ receptor $3, E 2 F 7$, phosphoserine aminotransferase 1 , spalt like transcription factor 1 , CEP55, KIF23, ret proto-oncogene, cell division cycle 25A, chromobox 2, cyclin E1, homeobox A10 (HOXA10), CHEK1 and claspin. GO and KEGG enrichment analysis for mRNAs co-expressed with lncRNAs and miRNAs indicated that the majority of the implicated genes are significantly involved in cell cycle-related biological processes mediating tumor cell proliferation. Another lncRNA MED4-AS1, which is targeted by hsa-mir-143 and hsa-mir-144, was overexpressed in the low-risk group. Several genes, including collagen type I $\alpha 1$ chain (COL1A1), COL5A2, T-box 18, potassium voltage-gated channel subfamily Q member 5 and $H O X A 10$, were predicted to be regulated by hsa-mir-143 and hsa-mir-144, and are clearly enriched in cell proliferation-associated GO terms. As studies on the roles and mechanisms of action of lncRNAs are in their infancy, functional interpretation of their co-expressed mRNAs within a ceRNA network is considered to be an effective computational strategy. The present study found that ABCA9-AS1 and MED4-AS1 may be involved in the 'skeletal system', 'protein binding', 'DNA binding', 'cell cycle' and 'p53 signaling pathway'. The skeletal system served a vital role in body support and movement. Meanwhile, collagen, as one component of the skeletal system, has been proven to promote tumor initiation and progression (31). It is widely accepted that the p53 tumor suppressor inhibits tumor growth by mediating cell-cycle arrest, apoptotic cell death and cellular senescence triggered by diverse cellular stresses (32). As a result, we hypothesize that dysregulation of these 9 lncRNAs associated LUAC prognosis contributes to the poor outcome of patients with LUAC by mediating known tumor-associated biological processes and pathways acting as ceRNAs regulating gene expression.

Currently, the TNM staging system is the most widely used system in predicting the survival of patients with LUAC. However, there are several limitations to the system. For example, not all stage III-IV patients experienced worse survival times compared with stage I-II patients, and patients who were in the same stage experienced variable survival times. Thus, the genetic predictive markers are required to assist doctors in forming more accurate estimates in clinical practice. In the present study, the identified 9-IncRNA signature showed prognostic value in LUAC patients. Even in the same pathological stage, the 9-IncRNA signature can classify patients into high- and low-risk groups with lncRNA expression level, suggesting that this lncRNA signature can improve the accuracy of survival prediction. Therefore, this result may aid doctors in selecting the corresponding therapeutic schedule for patients at different pathological stages, which can improve the overall survival of patients with LUAC.

However, there are certain limitations to the present study. First, the limited available lncRNA and miRNA expression profiles only identified a fraction of the lncRNAs that may be associated with LUAC prognosis. Second, the predictive value of IncRNA signatures remains to be verified by molecular and clinical experiments in future studies. Therefore, larger cohorts and experimental studies are required to validate this signature to further investigate the functional roles of LASiglnc-9 in LUAC prognosis.

In summary, the present study identified a 9-IncRNA signature that is closely associated with the tumor prognosis of patients with LUAC by use of lncRNAs profiles and construction of ceRNA networks, and by performing survival analysis. The present study not only indicates the predictive ability of lncRNA ceRNAs as potential biomarkers for LUAC diagnosis and prognosis, but also provides novel insight into the molecular mechanism underlying LUAC with further experimental validation.

\section{Acknowledgements}

Not applicable.

\section{Funding}

No funding was received.

\section{Availability of data and materials}

The datasets used and/or analyzed during the current study are available from the corresponding author on reasonable request. 


\section{Authors' contributions}

GF designed the study. XW, YD, BD and YF analyzed the data. XW and GF wrote the manuscript. All authors read and approved the final manuscript.

\section{Ethics approval and consent to participate}

Not applicable.

\section{Patient consent for publication}

Not applicable.

\section{Competing interests}

The authors declare that they have no competing interests.

\section{References}

1. Director's Challenge Consortium for the Molecular Classification of Lung Adenocarcinoma; Shedden K, Taylor JM, Enkemann SA, Tsao MS, Yeatman TJ, Gerald WL, Eschrich S, Jurisica I, Giordano TJ, Misek DE, et al: Gene expression-based survival prediction in lung adenocarcinoma: A multi-site, blinded validation study. Nat Med 14: 822-827, 2008.

2. Siegel RL, Miller KD and Jemal A: Cancer statistics, 2015. CA Cancer J Clin 65: 5-29, 2015.

3. Minna JD, Roth JA and Gazdar AF: Focus on lung cancer. Cancer cell 1: 49-52, 2002.

4. Sun S, Schiller JH and Gazdar AF: Lung cancer in never smokers-a different disease. Nat Rev Cancer 7: 778-790, 2007.

5. Ling H, Fabbri $M$ and Calin GA: MicroRNAs and other non-coding RNAs as targets for anticancer drug development. Nat Rev Drug Discov 12: 847, 2013.

6. Mattick JS and Makunin IV: Non-coding RNA. Hum Mol Genet 15 (Suppl 1): R17-R29, 2006.

7. Yu F, Yao H, Zhu P, Zhang X, Pan Q, Gong C, Huang Y, Hu X, $\mathrm{Su}$ F, Lieberman J and Song E: let-7 regulates self renewal and tumorigenicity of breast cancer cells. Cell 131: 1109-1123, 2007.

8. Kumar MS, Erkeland SJ, Pester RE, Chen CY, Ebert MS, Sharp PA and Jacks T: Suppression of non-small cell lung tumor development by the let-7 microRNA family. Proc Natl Acad Sci USA 105: 3903-3908, 2008.

9. Faraoni I, Antonetti FR, Cardone J and Bonmassar E: miR-155 gene: A typical multifunctional microRNA. Biochim Biophys Acta 1792: 497-505, 2009.

10. Nagano $T$ and Fraser P: No-nonsense functions for long noncoding RNAs. Cell 145: 178-181, 2011.

11. Prensner JR and Chinnaiyan AM: The emergence of lncRNAs in cancer biology. Cancer Discov 1: 391-407, 2011.

12. Weakley SM, Wang H, Yao Q and Chen C: Expression and function of a large non-coding RNA gene XIST in human cancer. World J Surg 35: 1751-1756, 2011.

13. Salmena L, Poliseno L, Tay Y, Kats L and Pandolfi PP: A ceRNA hypothesis: The rosetta stone of a hidden RNA language? Cell 146: 353-358, 2011.

14. Wee LM, Flores-Jasso CF, Salomon WE and Zamore PD: Argonaute divides its RNA guide into domains with distinct functions and RNA-binding properties. Cell 151: 1055-1067, 2012.

15. Wang J, Liu X, Wu H, Ni P, Gu Z, Qiao Y, Chen N, Sun F and Fan Q: CREB up-regulates long non-coding RNA, HULC expression through interaction with microRNA-372 in liver cancer. Nucleic Acids Res 38: 5366-5383, 2010.
16. Fan M, Li X, Jiang W, Huang Y, Li J and Wang Z: A long non-coding RNA, PTCSC3, as a tumor suppressor and a target of miRNAs in thyroid cancer cells. Exp Ther Med 5: 1143-1146, 2013.

17. Sui J, Li YH, Zhang YQ, Li CY, Shen X, Yao WZ, Peng H, Hong WW, Yin LH, Pu YP, et al: Integrated analysis of long non-coding RNAassociated ceRNA network reveals potential lncRNA biomarkers in human lung adenocarcinoma. Int $\mathrm{J}$ Oncol 49: 2023-2036, 2016

18. Sui J, Xu SY, Han J, Yang SR, Li CY, Yin LH, Pu YP and Liang GY: Integrated analysis of competing endogenous RNA network revealing lncRNAs as potential prognostic biomarkers in human lung squamous cell carcinoma. Oncotarget 8: 65997-66018, 2017.

19. Edge S, Byrd DR, Compton CC, Fritz AG, Greene F and Trotti A (eds): AJCC Cancer Staging Handbook: From the AJCC Cancer Staging Manual. 7th edition. Springer-Verlag, New York, NY, 2010.

20. Robinson MD, McCarthy DJ and Smyth GK: edgeR: A Bioconductor package for differential expression analysis of digital gene expression data. Bioinformatics 26: 139-140, 2010.

21. Shannon P, Markiel A, Ozier O, Baliga NS, Wang JT, Ramage D, Amin N, Schwikowski B and Ideker T: Cytoscape: A software environment for integrated models of biomolecular interaction networks. Genome Res 13: 2498-2504, 2003.

22. Alizadeh AA, Gentles AJ, Alencar AJ, Liu CL, Kohrt HE, Houot R, Goldstein MJ, Zhao S, Natkunam Y, Advani RH, et al: Prediction of survival in diffuse large B-cell lymphoma based on the expression of 2 genes reflecting tumor and microenvironment. Blood 118: 1350-1358, 2011.

23. Detterbeck FC, Boffa DJ and Tanoue LT: The new lung cancer staging system. Chest 136: 260-271, 2009.

24. Suzuki K, Yokose T, Yoshida J, Nishimura M, Takahashi K, Nagai K and Nishiwaki Y: Prognostic significance of the size of central fibrosis in peripheral adenocarcinoma of the lung. Anna Thorac Surg 69: 893-897, 2000.

25. Yanaihara N, Caplen N, Bowman E, Seike M, Kumamoto K, Yi M, Stephens RM, Okamoto A, Yokota J, Tanaka T, et al: Unique microRNA molecular profiles in lung cancer diagnosis and prognosis. Cancer Cell 9: 189-198, 2006.

26. Tay Y, Rinn J and Pandolfi PP: The multilayered complexity of ceRNA crosstalk and competition. Nature 505: 344-352, 2014.

27. Amer M, Elhefnawi M, El-Ahwany E, Awad AF, Gawad NA, Zada S and Tawab FM: Hsa-miR-195 targets PCMT1 in hepatocellular carcinoma that increases tumor life span. Tumour Biol 35: 11301-11309, 2014.

28. Fu MG, Li S, Yu TT, Qian LJ, Cao RS, Zhu H, Xiao B, Jiao CH, Tang NN, Ma JJ, et al: Differential expression of miR-195 in esophageal squamous cell carcinoma and miR-195 expression inhibits tumor cell proliferation and invasion by targeting of Cdc42. FEBS Lett 587: 3471-3479, 2013.

29. Yilaz Susluer S, Biray Avci C, Dodurga Y, Ozlem Dogan Sigva Z, Oktar N and Gunduz C: Downregulation of miR-195 via cyclosporin A in human glioblastoma cells. J BUON 20: 1337-1340, 2015.

30. Chabre O, Libe R, Assie G, Barreau O, Bertherat J, Bertagna X, Feige JJ and Cherradi N: Serum miR-483-5p and miR-195 are predictive of recurrence risk in adrenocortical cancer patients. Endocr Relat Cancer 20: 579-594, 2013.

31. Provenzano PP, Inman DR, Eliceiri KW, Knittel JG, Yan L, Rueden CT, White JG and Keely PJ: Collagen density promotes mammary tumor initiation and progression. BMC Med 6: 11, 2008.

32. Feng $\mathrm{Z}$ and Levine AJ: The regulation of energy metabolism and the IGF-1/mTOR pathways by the p53 protein. Trends Cell Biol 20: 427-434, 2010.

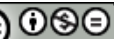

This work is licensed under a Creative Commons Attribution-NonCommercial-NoDerivatives 4.0 International (CC BY-NC-ND 4.0) License. 